surveyed was 8.3 years (range 0-3 months to 18 years). Numbers of at-risk patients by speciality were as follows: respiratory 33, oncology 28, neurology 34, rheumatology 12 , diabetes 24, nephrology 19 and immunology 3.83 (54.2\%) of at-risk children had received influenza vaccination in the past and 62 children (40.5\%) had received this season's influenza vaccine. $88.7 \%$ of these children had received their influenza vaccination in general practice, $9.7 \%$ in school, and $1.6 \%$ at Alder Hey. Out of the 62 at-risk children vaccinated against influenza this season, 20 (32.3\%) lived in a household in which all family members had been vaccinated. The most common reason given for children not receiving influenza vaccination was the lack of discussion over the vaccine with parents by health care professionals, in both primary and secondary care settings.

Conclusions The poor uptake of influenza vaccine for at-risk children attending Alder Hey reflects the global issue of inadequate influenza vaccination for such at-risk children. This brief survey provides further evidence that the importance of seasonal influenza vaccination is not being conveyed to the parents of at-risk children. Hospital specialists need to do more to assess the influenza vaccination status of their at-risk children. To facilitate this service improvement, the implementation of a hospital-based 'Immunisation Co-ordinator' role, working across both primary and secondary care providers, should be evaluated.

\section{G59(P) AN ABSCESS ON THE CHEST WALL - DON'T FORGET THE GREAT MASQUERADER}

S Kapoor, S Bandi. Paediatrics, Leicester Royal Infirmary, Leicester, UK

\subsection{6/archdischild-2018-rcpch.57}

Introduction Musculoskeletal tuberculosis (TB) occurs in 1\%$3 \%$ of patients with the disease. However cold abscess of the chest wall is rare and constitutes $1 \%$ to $5 \%$ of all cases of musculoskeletal TB. Moreover it is only described in cases of severe or disseminated form of the disease and not as the first presenting feature. Patients are often young and have no comorbidity. Diagnosis is often delayed because of the atypical presentation and lack of awareness. We describe a case of anterior chest wall mass as the first presenting feature of tuberculosis.

Case history A 15 year old Bangladeshi girl presented to us with a 2 month history of progressively increasing mass on the left anterior chest wall. She had a history of cough and intermittent fever over the preceding 2 weeks. There were no other systemic symptoms. Her immunisations were up-to-date and had received BCG at birth.

On examination she had a $6 \mathrm{~cm} \times 4 \mathrm{~cm}$ mass overlying the left 8th and 9th ribs, associated with an enlarged left supraclavicular lymph node. A chest $\mathrm{x}$-ray showed the mass eroding into the underlying 8th rib and a left hilar nodal shadow with a small left pleural effusion.

The patient underwent a diagnostic biopsy to exclude malignancy. This revealed a fluid filed centre and a significant amount of pus was drained. A post- biopsy chest X-ray showed a markedly enlarged pleural effusion. The patient's QuantiFERON test was positive $(16.05 \mathrm{iu} / \mathrm{ml})$. The fluid was sent for culture and sensitivity and this confirmed drug sensitive Mycobacterium tuberculosis. The patient was treated with standard Anti-Tubercular Therapy and had an uneventful recovery.

Conclusion Tuberculosis is a great masquerader and atypical presentations are known to occur. TB should be considered in children presenting with a chest wall mass even if they have been vaccinated with BCG. Other differential diagnoses include benign and malignant tumours. Prompt diagnosis and treatment of chest wall TB results in complete recovery and helps in preventing serious complications.

\section{G60(P) SMALL GROUP PAEDIATRIC DIETETIC SESSIONS FOR COW'S MILK PROTEIN ALLERGY (CMPA): ONE YEAR FOLLOW UP SURVEY}

SY Chang, N Rao, L Tomlinson, D Poulton, S Pavey. Paediatrics and Child Health, University Hospital of South Manchester, Manchester, UK

\subsection{6/archdischild-2018-rcpch.58}

Background/aim Small group dietetic sessions were held for parents and carers of patients with non-IgE mediated Cows Milk Protein Allergy (CMPA) to meet increasing demands. Initial feedback had been extremely positive with high participation and satisfaction rates. Participants found the group sessions informative, useful, and felt more confident in managing CMPA. We contacted the participants to obtain follow up feedback a year from the initial session, to evaluate the sustained effectiveness and confidence in parents managing their child's CMPA.

Method Parents and carers who attended the group dietetic sessions held between November 2015 and July 2016 were included. Feedback was obtained via a self-designed questionnaire using a Likert-type scale, rating several questions from 1 (least satisfied) to 5 (most satisfied), similar to the design of the initial questionnaire. We contacted the participants initially via telephone, and subsequently postal questionnaire with prepaid and labelled envelopes.

Results Of the 40 participants, we successfully obtained feedback from 13 participants. Majority of the participants agreed that the group sessions have been informative (mean score 4.8), felt confident in weaning their child on milk-free diet (mean score 4.8), and also reintroduction of cow's milk in diet (mean score 4.3).

$30 \%(n=4)$ preferred a one-to-one session. 38\% $(n=5)$ have contacted the dietitians via telephone after the initial session, and of these, 2 had requested further individual consultations. $69 \%(n=9)$ have attempted reintroduction of cow's milk in their child's diet using our local milk reintroduction guide. The mean age at first challenge was 14 months (age range 6 to 26 months), with average of two attempts. $31 \%(n=4)$ of the children have been successfully challenged and are managing well on a normal diet.

Conclusion We recognised the limitation in obtaining feedback via telephone and postal questionnaire, which resulted in the poor response rate. Overall, responses were positive and sustained a year on. Parents felt they had enough information and is confident in managing their child's CMPA. We have suggested emphasis on availability of follow up telephone or face-to-face consultations with the dietitians to allay parental anxiety. We will also pilot 'milk reintroduction' sessions to support and improve parental confidence in challenging their child with cow's milk at an appropriate age. 